\title{
Appareillage
}

\section{de mesure des concentrations spatiales dans un courant mixte d'air et d'eau}

\section{Apparatus for measuring spatial concentrations in a current of air and water mixed}

\author{
PAR J. BOUDAN
}

INGÉNEUR AU LABORATOMR DAUphINoIS D'HydraUligue

English synopsis, p. 354 .

\section{I. - But de l'appareillage}

Le principe de la mesure nécessite l'emploi d'une méthode de mesure électrique.

L'appareillage électrique doit traduire en une lecture précise les valeurs moyennes de la concentration spatiale en air du point de mesure.

Comme dans tout appareillage électrique ap-pliqué aux mesures hydrauliques, on doit distinguer :

- D'une part le capteur, dont le rôle est de transformer le phénomène hydraulique en une grandeur électriquement mesurable;

- D'autre part le système électrique traduisant cette grandeur, ou ses variations, en lecture ou en enregistrement.

\section{II. - Le capteur}

1) Propriétŕs électriouks

Le capteur est une sonde bifilaire qui a été décrite dans l'article de M. Halbronn.
Nous rappellerons simplement ses propriétés électriques.

La valeur de la concentration est une fonction du rapport de la résistance actuelle de la sonde à sa résistance en eaụ claire.

Il est plus simple de parler de la conductance de la sonde, plutôt que de sa résistance.

En posant :

$$
\mathrm{G}=\frac{1}{\mathrm{R}}
$$

on tire de la formule :

$$
\mathrm{C}_{\mathrm{s}}=\frac{\mathrm{R}-\mathrm{R}_{0}}{\mathrm{R}}
$$

Ia relation suivante :

$$
\frac{\mathrm{G}}{\mathrm{G}_{0}}=1-\mathrm{C}_{s}=\gamma_{s}
$$

avec:

$G$ conduclance actuelle,

$\mathrm{G}_{0}$ conductance en eau claire,

$\mathrm{C}_{s}$ concentration spatiale en air,

$\gamma_{s}$ concentration spatiale en eau. 
On voit que la conductance relative $\frac{G}{G_{0}}$ est égale à la concentration spatiale en eau.

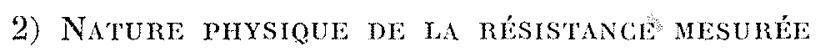

La résistance mesurée est une résistance électrolytique. La mesure n'a done pas de sens en courant continu (forces électromotrices de polarisation). On emploie done un courant alternatif de fréquence musicale.

Si la fréquence est trop ćlevée, les pertes en lignes ne seront plus négligeables. Si la fréquence est trop basse, l'impédance électrolylique n'est plus linéaire (harmoniques de courant) et le déphasage capacitif est important.

On choisit la fréquence de 6 kilocycles pour laquelle les pertes en ligne sont encore négligeables en module devant la consommation de la sonde. Elles se traduisent simplement par un faible déphasage ne troublant pas la mesure.

Même à cette fréquence, il y a apparition de polarisation (instabilité de l'impédance et destruction des surfaces) si les gradients de tension sont trop élevés au voisinage de la sonde. I a nature du métal a d'ailleurs une grosse importance.

Grâce aux précautions prises, la stabilité des caractéristiques est bonne, le déphasage of le taux de distorsion en harmoniques sont négligeables.

\section{III. - Système électrique de mesure}

\section{1) Caractéristroues du montage}

La concentration spatiale étant une grandeur fluctuante, la mesure n'aura d'intérêt que si on détermine une valeur moyenne dans le temps.

L'appareillage calculera électriquement la valeur moyenne du signal de sortie. Pour une fluctuation quelconque dans le temps, il n'y aura de relation entre la valeur moyenne du signal de sortie et la valeur moyenne de la concentration spatiale que dans le cas où le signal de sortie est une fonction linéaire de la concentration, au moins dans le domaine des fluctuations.

In effet, posons :

$$
u=f\left(\mathrm{C}_{s}\right)
$$

$u$ : signal de sortie.

Nous devons avoir la même relation entre les valeurs moyennes :

$$
u_{\text {moyen }}=f\left(\mathrm{C}_{s \text { moyen }}\right)
$$

Cette égalité n'est vérifiée que si f est une fonction linéaire de $\mathrm{C}_{s}$, ou, dans le cas, non rencontré en pratique, ou $\mathrm{C}_{s}=$ constante.

Ón voit que la caractéristique du montage électrique doit être de délivrer un signal de sortie fonction lineaire de la concentration spatiale.

Nous allons décrire le « montage linéaire en admittance » employé d'ailleurs dans différents systèmes de mesure de ce type (mesure de houle, mesure de salinité).

Considérons une impédance complexe variable. On place en série une seconde impédance fixe $a$, de module inférieur au quinzième de la valeur minimum du module de l'impédance variable, et en quadrature avec celle-ci (fig. 1).

Le module de l'impédance série diffère au maximum de $0,22 \%$ du module de l'impédance variable $\mathrm{X}_{s}$.

Si le système est alimenté à tension fixe $U$, le courant dans l'impédance totale, donc la tension aux bornes de l'impédance fixe $a$, varient linéai-

$$
\begin{gathered}
x=|x| \mid \alpha \\
a=|a| \frac{\mid \alpha+\frac{n}{2}}{\sqrt{1+\left(\frac{a}{|x|}\right)^{2}}} \\
\left.(x+a)=|x| \sqrt{n^{2}}\right) \\
\sim|x|\left(1+\frac{1}{2}\left(\frac{|a|}{|x|}\right)^{2}\right) \\
\sim|x| \text { à } 0,22 \% \text { au minimum }
\end{gathered}
$$

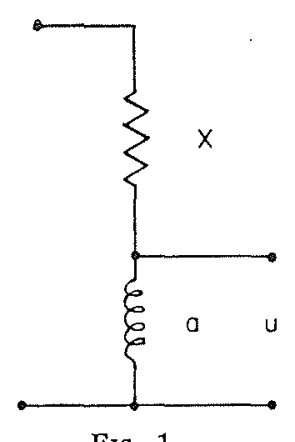

FIG. 1. rement avec l'admittance variable, à $0,22 \%$ près au maximum.

L'affaiblissement de tension dans un tel montage est de 15 . Il serait de 450 avec un montage à résistance série de même précision, d'où un gain de 30 sur le coefficient d'amplification.

De plus, on peut choisir la nature de l'impédance fixe de manière à filtrer les parasites industricls que la sonde rencontre sur le modèle, c'est-à-dire une impédance inductive à faible angle de perte, répondant aux conditions de module et de phase données ci-dessus.

La tension est stabilisée aux bornes du systìme par une capacité de faible impédance.

Le " montage linéaire en admittance » transforme donc la variation de l'admittance de la sonde en variation de tension. La tension est proportionnelle à l'admittance.

Nous admettons que le module de l'admittance est proportionnel à la conductance, ce qui se vérifie avec une grande précision. L'argument de la sonde reste très faible et approximativement constant. 
La tension aux bornes de a est done en définilive proportionnelle à la conductance de la sonde, donc à la concentration en cau.

Pour obtenir une tension proportionnelle à la concentration spatiale en air, nous montons en pont avec le systeme ci-dessus, une seconde branche formée d'impédances de même nature que dans la première branche : une inductance à faible perte et une résistance variable, shuntée par une capacité variable.

La diagonale de mesure étant à très haute impédance d'entrée (grille d'amplificateur), il n'y a pas d'interaction entre les deux branches, la tension d'alimentation dant constante.

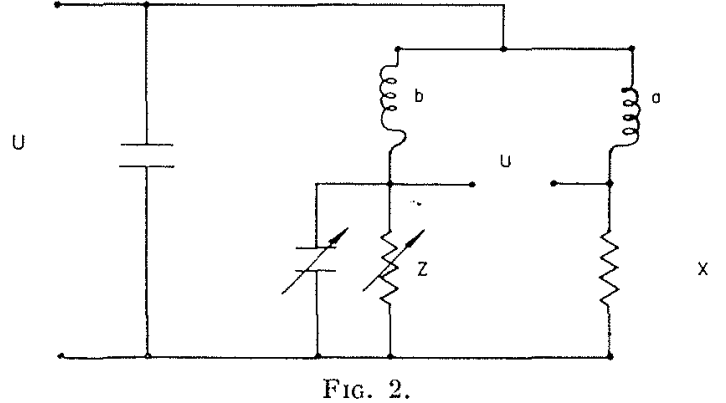

Un calcul complet nous donne pour valeur de la tension dans la diagonale de mesure (fig. 2) :

$$
\frac{u}{\mathrm{U}}=\frac{a}{a+\mathrm{X}}-\frac{b}{b+\mathrm{Z}}
$$

$a$
$b$ inductance fixe,

$\mathrm{X}$ impédance variable,

$Z$ impédance d'opposition.

Nous pouvons écrire, à $0,2 \%$ près, la relation cntre les modules :

$$
\frac{u}{\mathrm{U}}=a \mathrm{G}-\mathrm{A}
$$

Le pont est équilibré en eau claire, done :

$$
a \mathrm{G}_{0}-\mathrm{A}=0
$$

et :

$$
\frac{u}{\mathrm{U}}=a \mathrm{G}_{0}\left(\frac{\mathrm{G}}{\mathrm{G}_{\mathrm{x}_{0}}}-1\right)
$$

enfin :

$$
\frac{u}{\mathrm{U}}=a \mathrm{G}_{0} \mathrm{C}_{s}
$$

Remarquons que, la sonde étant étalonnée avec le montage de mesure, l'erreur de mesure sur la valeur moyenne est inférieure à la valeur donnée ci-dessus.

Calculons cette erreur dans quelques cas simples de fluchuations.

Supposons une fluctation lincaire dans le temps, en dents de scie ou en triangle, entre 0 et 1 de concentration. L'erreur sur la valeur moyenne est alors de :

$$
e=0,037 \% \text {. }
$$

Supposons une fluctuation par tout ou rien (en signaux carrés) entre 0 et 1 , de valeur moyenne 0,5 . L'erreur sur la valeur moyenne est alors :

$$
e=0,055 \%
$$

Ces deux cas sont des cas défavorables.

\section{2) Appareitlage Électronioue}

La tension sinusoïdale basse fréquence SKC est fournie par un oscillateur à battements, à faible taux de distorsion d'harmoniques et à alimentation stabilisce, d'une excellente stabilité en fréquence et en niveau de sortie.

Le nont d'impédance est alimenté à travers un transformateur à écran, pour séparer les masses du générateur et du pont.

L'amplificateur a une masse commune au générateur, mais isolée de la masse du modèle, de manière à éviter les fuites de courant de la sonde à la masse.

L'amplificateur comporte trois étages de gain, a forte contre-réaction par la grille pour le premier étage, et par la cathode pour les étages suivants. Te gain total est de 80 décibels. Le gain de ces étages ne dépend que pour quelques pour cent des caractéristiques des lampes, ce qui assure une stabilité excellente et une remarquable linéarité pour un fort niveau de sortie. De plus, le gain est dans une large bande, indépendant de la fréquence. Cette dernière condition a son importance. On sait en effet que la modulation d'amplitude se traduit par une modulation de frécuence de largeur de bande double de la fréquence de modulation, qui peut afteindre quelques centaines de cycles/seconde.

Le dernier étage est un étage de puissance de gain, le signal étant pris sur la cathode.

Il est suivi par une détection parallèle et par un filtre à résistance capacité donnant la valeur moyenne du signal de sortie. En modifiant les cellules du filtre, on peut prendre la valeur moyenne sur l'intervalle de temps désiré, le temps nécessaire pour obtenir une lecture correcte restant assez faible. 
La sortie du filtre est reliée à la grille d'un tube sur la cathode de laquelle se trouve la premiere borne d'un milliampèremètre.

\section{3) LeCTURE}

Pour lire la tension de sortie avec une précision constante quelle que soil la coneentration, on emploie une méthode d'opposition.

La seconde borne du milliampéremetre est connectée à la cathode d'un tube dont la grille est reliée au curseur d'une boîte jumeléc à quatre dieades (fig. 3).

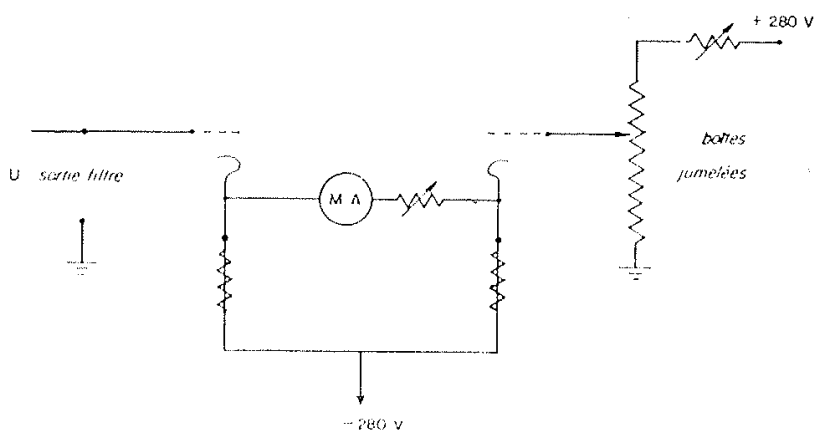

Fig. 3.

Apres avoir équilibré le pont en cau claire, on débranche la sonde, ce qui est équivalent à une concentration de $100 \%$ en air.

On écrit 100 sur la boìte jumelée et on riggle le débit dans cette boite à l'aide d'un rhéostat série pour égaliser la tension de sortie et la tension d'opposition, ce qui amene le milliampèremètre à zéro.

En cours de mesure, on manipule la boite pour amener le milliampèremètre à zéro, en accroissant la sensibilité en approchant de l'équilibre.

On lit alors sur la boite la concentration en air en pour cent.

\section{IV. - Erreur et fidélité}

\section{1) Erreurs dues a la sonde}

M. Halbrony a discuté ces erreurs dans son article : la formule théorique se vérifie avec une bonne précision. L'erreur d'inclinaison est faible. L'erreur de température s'élimine par des tarages répétés. Celte erreur est surtout importante pour les petites concentrations, car elle entraîne un déréglage du zéro du pont en eau claire.
En tenant compte de la température, la formule (7) s'écrit :

$$
\frac{u}{\mathrm{U}}=a \mathrm{G}(0)-a \mathrm{G}_{0}\left(0_{0}\right)
$$

$G(0)$ étant la conductivité actuelle à la concentration $c_{s}$ et $\mathrm{C}_{0}\left(0_{0}\right)$ la conductivité en cau claire.

(10) peut s'écire :

$$
\frac{u}{\mathrm{U}}=a G\left(0_{0}\right)+a d \mathrm{x}-a\left(\mathrm{x}_{0}\left(0_{0}\right)\right.
$$

el d'apres (10):

$$
\frac{u}{\mathrm{U}}=a \mathrm{G}_{0} \mathrm{C}_{\mathrm{s}}+a \Delta \mathrm{G}=a \mathrm{G}_{0}\left(\mathrm{C}_{\mathrm{s}}+\frac{\Delta \mathrm{G}}{\mathrm{G}_{0}}\right)
$$

or :

$$
\frac{\Delta \mathrm{G}}{\mathrm{G}_{01}}=\frac{\Delta \mathrm{G}}{\mathrm{G}} \frac{\mathrm{G}}{\mathrm{G}_{0}}=e_{k}\left(1-\mathrm{C}_{\mathrm{k}}\right)=e_{i,} \% \mathrm{~S}
$$

$e_{\text {it }}$ ćant la variation relative de conductivité due a la température.

$$
\frac{u}{U}=a C_{01}\left|C_{x}+e_{\theta}\left(1-C_{x}\right)\right|
$$

On voit qu'une variation de température introduit une erreur absolue proportionnelle à la concentration en eau. Le coefficient de température étant de $3 \%$ par degré environ, une variation d'un dixiène de degré, pour une concentration de $1 \%$, introduit une erreur d'environ 0,3 point de concentration.

On doit done surveiller l'évolution de la température avec un thermometre de grande précision et apporter les corrections à la mesure, ou mieux procéder à un nouveau tarage. Cette opération se fait très rapidement, la sonde pouvant être replacée instantanément en eau claire grâce à une petite coupe placant la sonde en un milieu dégazé.

Au point de vue stabilité des caractéristiques électriques, la sonde a donné toutes satisfactions grâce aux soins apportés à sa réalisation mícanique.

\section{2) Erreyrs dues aU montage}

Nous avons estimé ci-dessus lerreur introduile dans la mesure de la valeur moyenne par le montage. Elle est inférieure à $0,06 \%$.

La linéarité de l'amplificateur ćtant de l'ordre de $0,1 \%$, l'erreur tolale sur la valeur moyenne est de l'ordre de $0,1 \%$.

Les décades de lecture sont à la précision de $0,2 \%$

L'erreur due au voltmetre de lecture est inférieure à $0,1 \%$. 
En pratique, pour réaliser la condition de linéarité du montage pour les plus petites concentrations, on doit donner au pont un léger déséquilibre en module en réglant la valeur de la résistance variable un peu en dessous de la valeur d'équilibre.

Ce réglage a pour but d'éliminer la zone de minimum flou dû au faible résiduel d'harmoniques et aux capacités parasites du montage.

Il a d'autre part l'avantage d'éliminer le résiduel de détection.

Il a, par contre, l'inconvénient d'augmenter légèrement l'erreur absolue de température et d'introduire une erreur absolue due à l'instabilité électronique.

Le déséquilibre de base est de l'ordre du point de concentration. On voit que l'augmentation de l'erreur de température est négligeable. Au lieu de :

$$
e_{*}\left(1-\mathrm{C}_{s}\right)
$$

elle devient :

$$
e_{3}\left(1-\mathrm{C}_{s}+0,01\right)
$$

D'autre part, pour une instabilité électronique de $5 \%$, l'erreur absolue introduite est de 0,05 point de concentration.

\section{3) Erreurs dues a l'instabilité} DE L'APPAREILLAgE ÉLECTRONIQUe

Pour une valeur donnée de l'admittance de la sonde, le signal de sortie est fonction : de la fréquence, du niveau de sortie BF et dụ gain de l'amplicateur.

D'autre part, la lecture est fonction de la tension d'opposition, qui est d'ailleurs la haute tension générale d'alimentation de l'appareillage.

Les hautes tensions de 280 volts sont obtenues à partir de sources à 550 volts et de stabilisateurs au néon. On obtient ainsi une stabilisation supérieure au demi pour cent.

L'ensemble de l'appareillage est d'ailleurs alimenté par un transformateur à fer saturć stabilisant le réseau à $2 \%$.
Remarquons que la variation de Ia tension d'opposition est dans le même sens et compense en partie la variation de tension de sortie du générateur et de gain de l'amplificateur. Après une mise en route d'une demi-heure, on obtient une stabilité de l'ordre du demi pour cent, ce qui rend négligeable l'erreur absolue signalée ci-dessus.

L'instabilité de l'électronique se traduit done par une erreur relative inférieure à $0,5 \%$. Cette erreur peut être compensće par un tarage très simple en cours de mesure puisqu'il suffit simplement de débrancher la sonde.

\section{4) Conclusion sur les erreurs}

L.es erreurs se résument donc :

- Une erreur systématique globale due aux monlages et à l'appareillage inférieur à $0,4 \%$ en valeur relative.

- Une infidélité due à l'instabilité de l'électronique, que l'on peut compenser par de fréquents tarages. Cette erreur est en valeur relative inférieure à $0,5 \%$.

- Une erreur absolue due à la température, que l'on doit compenser par une lecture précise des températures et de fréquents tarages.

- Des erreurs accidentelles dues à la sonde : inclinaison, approche des parois, discutées dans l'article de M. Halbronn.

\section{V. - Conclusion}

La sonde bifilaire et l'appareillage de mesure forment un tout permettant de mesurer d'une manière précise une grandeur difficilement saisissable jusqu'ici.

Cet ensemble s'est révélé en pratique bien adapté à la mesure et d'un maniement commode en cours de campagne. La précision des résultats obtenus est satisfaisante, moyennant certaines précautions expérimentales. 